\title{
Environmental system effects when including scrap preheating and surface cleaning in steel making routes
}

\author{
Marianne Östman ${ }^{1, *}$, Katarina Lundkvist ${ }^{1}$, Mikael Larsson ${ }^{1}$ \\ ${ }^{1}$ Swerea MEFOS, Luleå, Sweden \\ *Corresponding author. Tel: +46 920201900, Fax: +46 920255832, E-mail: marianne.ostman@swerea.se
}

\begin{abstract}
The main part of the steel manufactures producing alloyed steel use scrap as an essential raw material. To increase the corrosion resistance of steel a coating is often applied. The share of steel being coated and galvanized is today globally increasing, which is resulting that the amount of scrap with different types of coatings are increasing. This result in that more of the scrap used in steel making is contaminated with for example Zinc and organics. Scrap preheating is a known method for reduction of energy use in steelmaking. However due to environmental restrictions a widespread implementation of scrap preheating have not been achieved in the steel industry. Combined surface cleaning and scrap preheating is a way to handle the problem that coatings give rise to in the melting cycle and is a new concept suggested and developed at Swerea MEFOS. The pre-treated scrap (cleaned and preheated) is charged hot into the oxygen converters with direct savings of energy, as the demand of hot metal from the blast furnace decreases. The method opens for the possibility to widen the scrap base and to melt complicated scrap. Since the preheating process is a standalone solution, a large number of unwanted chemical components are removed prior melting resulting in that the dust generated from the melting process is easier to recover. In this paper the system effect of introducing a preheating and surface cleaning process for scrap in a Blast Furnace (BF) and oxygen converter (BOF) steelmaking route is analyzed according to energy and $\mathrm{CO}_{2}$ emissions. The system analysis shows that surface cleaning of scrap makes it possible to use shredded scrap and ASR (automotive shredder residue) or other combustible waste to replace fossil fuels. The results from the analysis demonstrates that implementing surface cleaning leads to increased possibilities for recycling of otherwise non-recyclable material. The system model shows the interaction between different processes, which gives an overview picture including the whole steel making route. The model is used to investigate changes in process conditions from making use of shredded scrap and ASR as input material in the steel making process. The implementation of surface cleaning and preheating lead to both increased possibilities for recycling of scrap, and more efficient energy use in the steelmaking routes.
\end{abstract}

Keywords: BOF, Scrap preheating, Surface cleaning, optimization, ASR

\section{Introduction}

Currently, the blast furnace (BF), basic oxygen furnace (BOF) route is the dominating processes for iron ore steel making. The steel is produced from the hot metal produced in the $\mathrm{BF}$ by treatment in a BOF converter. In the converter process a surplus heat is generated during the oxygen blowing which is utilized for scrap melting. 15-20\% of the iron raw material input to the BOF is originated from scrap. Liquid steel (LS) from the converter is sent to an after-treatment station where the final adjustment of the steel analysis and temperature is performed before the casting procedure. Energy and material efficiency in a process system is closely linked together. A higher recycling rate and a minimization of landfill is energy efficient, since it decreases the demand of virgin materials.

The main part of the steel manufactures producing alloyed steel use scrap as an essential raw material. To increase the corrosion resistance of steel a coating is often applied. The share of steel being coated and galvanized is today globally increasing, which is resulting that the amount of scrap with different types of coatings are increasing. This result in that more of the scrap used in steel making is contaminated with for example Zinc and organics. In this paper the system effect of introducing a preheating and surface cleaning process for scrap in a Blast Furnace and oxygen converter (BOF) steelmaking route is analyzed according to energy and $\mathrm{CO}_{2}$ emissions. 
Scrap preheating is a known method for reduction of energy consumption for melting. However due to hazardous components generated in the exhaust, a widespread implementation of scrap preheating have been interfered due to the costly treatment required. A standalone combined surface cleaning and scrap preheating is a way to handle the problem that coatings on the scrap give rise to. This is a new concept suggested and developed at Swerea MEFOS [1]. The pre-treated scrap (cleaned and preheated) is charged hot into the oxygen converters with direct savings of energy, as the demand of hot metal from the blast furnace decreases. The method opens for the possibility to widen the scrap base and to melt complicated scrap. Since the preheating process also applies surface cleaning a large number of unwanted chemical components are removed prior melting resulting in that the dust generated from the melting process is easier to recover. [1-2]

\subsection{Objective of the study}

This study analyzes the system effect of introducing surface cleaning of scrap in a BF-BOF steel making route. An optimization model highlights the possibilities regarding cost and $\mathrm{CO}_{2}$ emission. The model is used to do analysis on operation practice, scrap usage and cost. The intention of the study is to investigate the changes in the process system, when using shredded scrap in the scrap mix.

\section{Methodology}

The method used in this work is based on the MIND method [3]. The study is made in an optimization model of an integrated steel mill, where the process is described as a network of nodes (sub-processes) which are connected by energy and material flows. The potential of this method is that is enables a simultaneous representation of the total industrial system, and that is makes it possible to optimize the whole system, in contrast to the optimization of each subprocess individually. The optimization model of one integrated steel plant has been further developed with the requirements for scrap preheating [4-5]. The model used in this work is based on an existing energy optimization model designed for the iron and steel industry [6-8].

\subsection{The optimization model}

\subsubsection{Objective function}

This study uses two objective functions which are defined in the model as raw material and energy costs and $\mathrm{CO}_{2}$ emission. Generally the objective function is imbedded within the optimization model but can in mathematical terms be written as follows.

$$
\min z=\sum_{i=1}^{n} c_{i} x_{i}
$$

where $z$ is the objective function for the minimization problem. It could be $\mathrm{CO}_{2}$ emission and cost, depending on what objective is set for the optimization. $x$ is studied variables and $c$ is coefficients set for the corresponding objective function and depends on which objective function. The coefficients set for the corresponding objective functions are shown in Table 1 . 
Table 1. Coefficients used for different objective functions.

\begin{tabular}{lccc}
\hline & Unit & Cost (SEK) & $\mathrm{CO}_{2}$ emission (ton) \\
\hline Iron ore pellet & ton & 675 & 0.051 \\
Lime stone (wet) & ton & 128 & 0.856 \\
Mn ore & ton & 488 & - \\
Quartz & ton & 180 & - \\
Lime stone & ton & 124 & 0.856 \\
Dolomite Lime & ton & 600 & 0.466 \\
Raw Dolomite Lime & ton & 263 & 0.931 \\
FeSi & ton/ton RS & 4875 & - \\
CaC 2 & ton & 3225 & - \\
Coal and coke & ton & $293-331$ & $2.492-3.064$ \\
External coke & ton & 1950 & 3.744 \\
Pulverized coal injection & ton & $205-525$ & $2.488-2.916$ \\
External scrap & ton & 2300 & - \\
Shredded scrap & ton & 2000 & - \\
BOF sludge & ton & - & - \\
Oil & MWh & 5550 & 3.126 \\
External energy & MWh & - & 0.6 \\
Excess coke & ton & -1950 & -3.744 \\
Benzene & ton & -1600 & -3.287 \\
Sulphur & ton & -40 & - \\
Tar & ton & -1300 & -3.349 \\
\hline
\end{tabular}

In Table 1, the costs analysis is limited to raw material and energy and thereby not including the cost for energy and landfill of material. Furthermore credits are made in the model for byproducts. Market values for the different raw materials and energy sources fluctuate whereby the costs for raw materials and energy used in the model is mean values calculated over a conjuncture cycle. Values of costs are estimations based on figures from The London Bullion Market Association.

\subsubsection{Boundary conditions and limitations}

To make sure to get reasonable results, some necessary boundary are introduced. The boundary conditions, which can describe variations in the system, maximum and minimum for various variables can be expressed as follows.

$x_{i} \leq b_{i}, \quad i=1, \ldots, n$

where the $x_{i}$ variable could be the corresponding flow variables, and the boundaries $b_{i}$, are the corresponding restrictions. As a boundary condition the production of prime slabs is fixed in the model. The BOF charges has limitations, such as hot metal, pig iron, slag binders and scrap are set to the production parameters of liquid steel, to ensure the right quality for the final products.

\subsection{Model description}

In the iron and steel industry optimization models considering effects on the entire production process chain have been used for investigations of performance of different aspects like energy consumption, $\mathrm{CO}_{2}$ emission, costs and environmental issues [4-8]. The system boundary of the whole system is to casted steel slab, the boundary of this study is to RS (Raw 
Steel) from the BOF converter. The process units included are coking plant, blast furnace (BF), basic oxygen furnace (BOF), and continuous casting (CC), oxygen plant, and lime furnace, etc. [9]

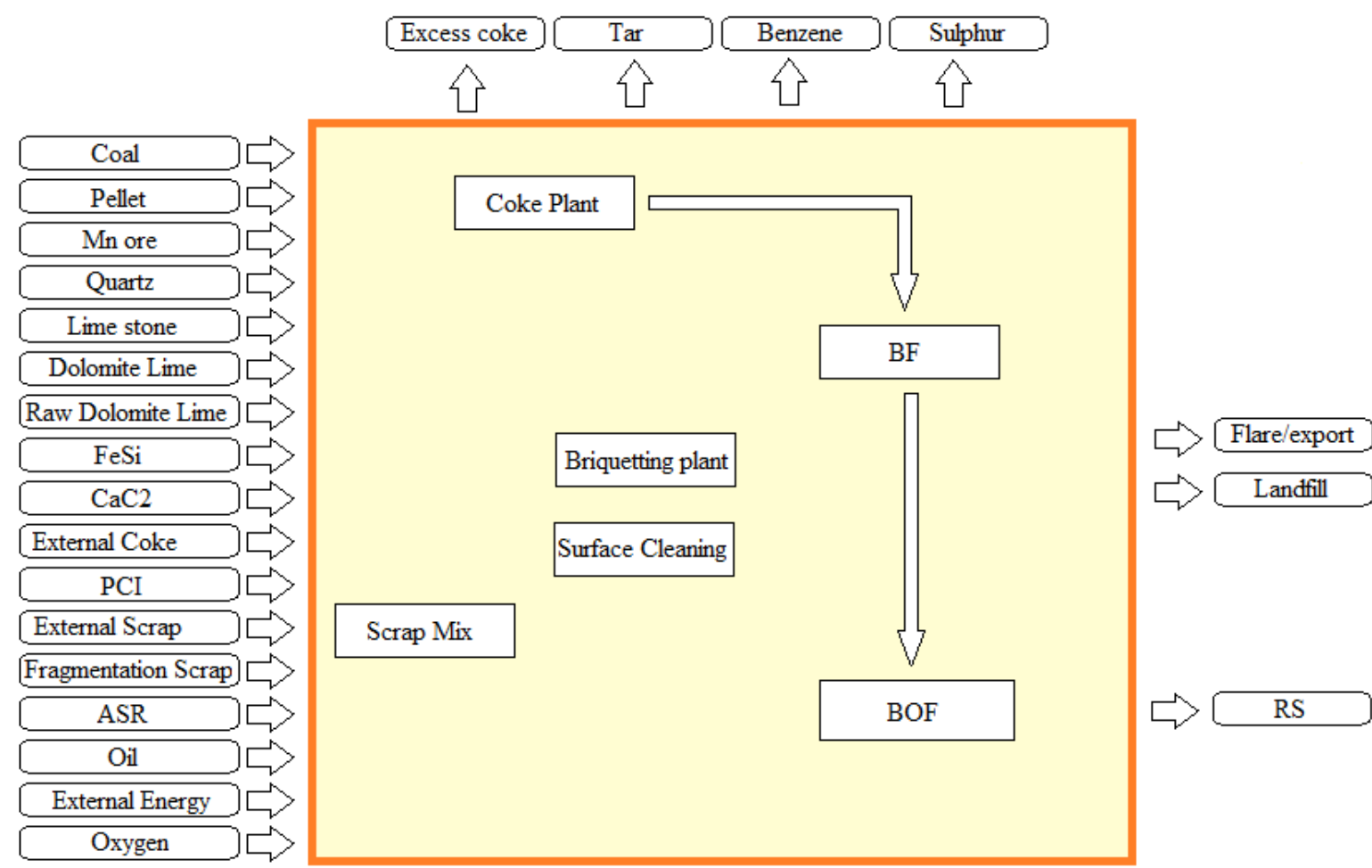

Fig. 1. Schematic outline of the modeled system.

The blast furnace is a very complex process and thus difficult to model. In this study different operational conditions for the blast furnace, which are feasible are defined. The model chooses and combines between these to overcome this problem. The prepared model is consisting of modules of process steps connected by mass and energy balances.

\subsubsection{Operating conditions and modeling cases}

The BF module is simulating the hot metal production in a Swedish BF with potential changes in briquette recipe and charged amounts. The BOF production process is described in the model by the mass and energy balance of the process. Different options can be optimized regarding the raw materials used due to the chemical composition. The coarse fraction of the generated BOF sludge is recycled through briquettes charged into the BF. The fine fraction of sludge from the BOF is normally land filled however it may be recycled in briquettes provided the zinc content is reduced sufficiently [10]. Including the scrap preheating unit in the system contributes to the scrap usage efficiency by the charging of preheated scrap. The introduction of the scrap surface cleaning unit in the model makes the use of shredded scrap as a scrap source to the BOF viable as it reduces the Zinc content in the scrap input to the BOF to zero. Scrap cleaning and preheating is performed on all the scrap charged to the BOF when the pre-treatment operation is implemented. Since the exhaust problem with the pretreatment of scrap roughly identical with the combustion of many types of polluted fuels, Therefore the preheating process needs an advanced gas cleaning facility which gives that fossil fuel could be replaced with organic rest material. In this study ASR (automotive shredder residue) has been chosen as fuel in the preheating process since it has a good heating value and can be delivered in enough quantity to be interested for industrial use. Zinc sources 
to the BOF sludge and dust in the model is the external scrap and the shredded scrap. Sources of Zinc to the BF come from the iron ore pellets and briquettes. The possible integration and effect of the preheating and surface cleaning operation is analyzed in five cases compared against a reference case, Table 2. The input of scrap to the BOF is limited to $17 \%$ in the reference case, case 1 and 2, since it is a reasonable amount. To ensure that the use of internal scarp is preferred, the external scrap use is limited to maximum $5 \%$ of the scrap charge into the BOF. The optimization cases have a higher degree of freedom on the BF operation and on the raw material input in the BOF.

Table 2. Case study.

\begin{tabular}{ll}
\hline Case & Comments \\
\hline Reference Case & BF operation with briquettes \\
Case 1 & $\begin{array}{l}\text { BF operation with briquettes, scrap pretreatment implemented, otherwise } \\
\text { as the Reference Case }\end{array}$ \\
Case 2 & BF operation without by-product briquettes, otherwise as the Ref. Case \\
Case 3 & Optimization of cost, Scrap preheating/surface cleaning available \\
Case 4 & Optimization of $\mathrm{CO}_{2}$, Scrap preheating/surface cleaning available \\
\hline
\end{tabular}

\section{Results}

\subsection{System optimization}

Data from the system modeling of production and differences in the analyzed cases is illustrated in Table 3. Regarding the coke making there are only minor differences between the reference production and the five different cases. The production rate of coke is the same in all five cases. Small change in coal mix volatile matter and ash result in small increase in COG production compared to the reference case. To increase recycling inside the steel plant by-product briquettes are used. Data from the system model of BF operation show that the $\mathrm{HM}$ (Hot Metal) production is higher in the reference case and in case 2 which is using no briquettes. Increased use of briquettes is positively affects the cost- and CO2 emission optimized cases by the lower lime stone and pellet consumption in the BF.

\subsubsection{Iron ore pellet consumption}

The use of iron ore pellets in the analyzed cases vary. Iron ore pellets has a $\mathrm{CO}_{2}$ emission burden of 0.051 ton, as a comparison to scrap which has zero, se Table 1 , since scrap is a recycled material origin from iron ore. In case 2 where no briquettes are produced the major amount of pellets is being utilized due to less recycling of iron bearing by-products. In the case 4, where $\mathrm{CO}_{2}$ emission is the optimization target, the lowest amounts of pellets are used.

\subsubsection{Scrap consumption}

Producing steel from scrap is better from an environmental point of view since the energy demand for producing steel from iron ore is more than twice the energy required in scrap based production. Scrap usage for the cases is illustrated in Fig. 2. Shredded scrap may only be utilized in the cases when surface cleaning is implemented. The Reference case, case 1 and 2 has a limitation on the scrap usage to a maximum load of $17 \%$, where maximum of external scrap is $5 \%$ in the BOF. The largest amount of scrap utilized is in case 4 , which is the $\mathrm{CO}_{2}$ emission optimization case. Since energy use and $\mathrm{CO}_{2}$ emissions from integrated steelmaking is closely related, increased scrap utilization results in less $\mathrm{CO}_{2}$ emissions. 
Table 3. Optimization results coke making, Blast furnace and BOF route.

\begin{tabular}{|c|c|c|c|c|c|}
\hline & Ref. & Case 1 & Case 2 & Case 3 & Case 4 \\
\hline \multicolumn{6}{|l|}{ Coke plant } \\
\hline Coke production $(\mathrm{t})$ & 84.5 & 84.5 & 84.5 & 84.5 & 84.5 \\
\hline COG production ( $\mathrm{GJ} / \mathrm{t}$ coke) & 8.4 & 8.4 & 8.4 & 8.4 & 8.4 \\
\hline Coke yield, dry (t coke/t coal) & 1.287 & 1.287 & 1.287 & 1.287 & 1.288 \\
\hline Excess coke (t) & 0.00 & 0.00 & 0.00 & 0.00 & 0.00 \\
\hline Ash (\%, dry basis) & 9.12 & 9.12 & 9.12 & 9.12 & 9.14 \\
\hline Volatile matter (\%, dry basis) & 25.60 & 25.60 & 25.60 & 25.60 & 25.66 \\
\hline \multicolumn{6}{|l|}{$\mathrm{BF}$} \\
\hline HM production (t/h) & 250.1 & 244.4 & 250.1 & 246.6 & 246.4 \\
\hline BF slag production (t/t HM) & 0.162 & 0.162 & 0.156 & 0.164 & 0.164 \\
\hline Pellet (kg/t HM) & 1356 & 1356 & 1394 & 1339 & 1340 \\
\hline Coke (kg/t HM) & 320 & 320 & 330 & 317 & 317 \\
\hline PCI (kg/t HM) & 141 & 141 & 141 & 141 & 141 \\
\hline Lime stone (kg/t HM) & 30 & 30 & 46 & 20 & 20 \\
\hline By product briquettes (kg/t HM) & 59 & 59 & 0 & 84 & 82 \\
\hline BOF slag $(\mathrm{kg} / \mathrm{t} \mathrm{HM})$ & 46 & 46 & 46 & 46 & 46 \\
\hline Other (kg/t HM) & 4 & 4 & 5 & 4 & 4 \\
\hline Blast $\left(\mathrm{kNm}^{3} / \mathrm{t} \mathrm{HM}\right)$ & 0.98 & 0.98 & 0.98 & 0.98 & 0.98 \\
\hline BFG (GJ/Nm3 blast) & 1.13 & 1.13 & 1.13 & 1.15 & 1.15 \\
\hline COG (GJ/Nm3 blast) & 0.71 & 0.71 & 0.71 & 0.72 & 0.72 \\
\hline \multicolumn{6}{|l|}{ BOF } \\
\hline $\mathrm{RS}(\mathrm{t} / \mathrm{h})$ & 257.5 & 257.5 & 257.5 & 257.5 & 257.5 \\
\hline BOF slag $(\mathrm{t} / \mathrm{h})$ & 0.08 & 0.08 & 0.08 & 0.08 & 0.08 \\
\hline $\mathrm{HM}(\mathrm{kg} / \mathrm{t} \mathrm{RS})$ & 879 & 859 & 879 & 867 & 866 \\
\hline Pellets (kg/t RS) & 0 & 8 & 0 & 11 & 10 \\
\hline Scrap mix (kg/t RS) & 175 & 188 & 175 & 179 & 225 \\
\hline Lime (kg/t RS) & 27 & 26 & 27 & 26 & 25 \\
\hline Dolomite lime (kg/t RS) & 23 & 23 & 23 & 23 & 22 \\
\hline Raw dolomite lime (kg/t RS) & 4 & 4 & 4 & 4 & 4 \\
\hline $\mathrm{O}_{2}$ blowing $\left(\mathrm{Nm}^{3} / \mathrm{t} \mathrm{RS}\right)$ & 50.0 & 47.7 & 50.0 & 47.7 & 47.6 \\
\hline Scrap preheating on/off (1/0) & 0 & 1 & 0 & 1 & 1 \\
\hline $\mathrm{O}_{2}\left(\mathrm{Nm}^{3} / \mathrm{t} \mathrm{scrap}\right)$ & 37 & 37 & 37 & 37 & 37 \\
\hline ASR (kg /t scrap) & 32 & 32 & 32 & 32 & 32 \\
\hline
\end{tabular}

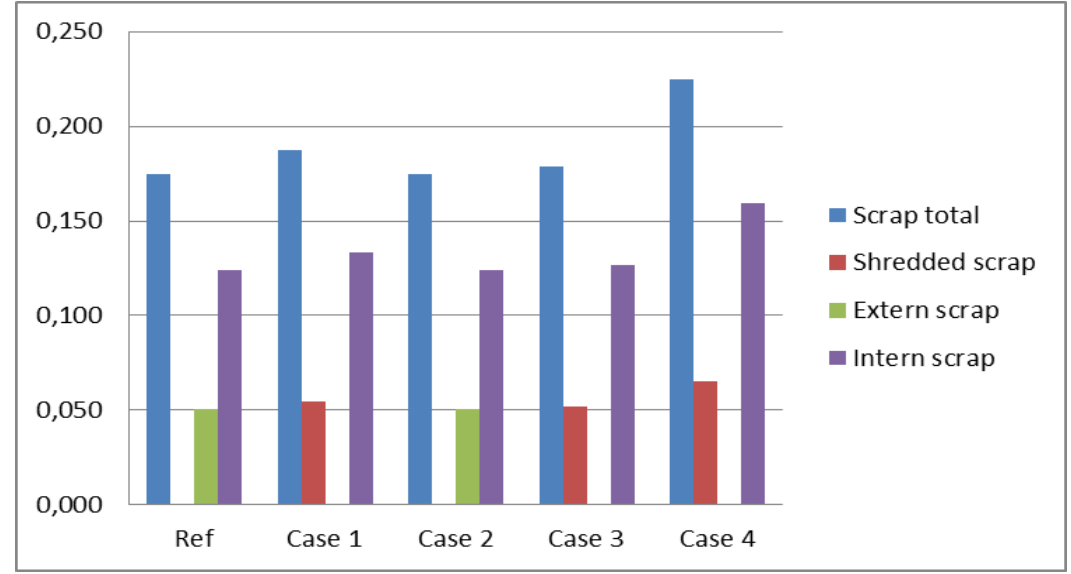

Fig. 2. Scrap consumption in the different cases, unit $t / t$ RS. 


\section{Discussion and Conclusions}

\subsection{Results from the objective function analysis}

Table 4. Results from the objective functions.

\begin{tabular}{ccc}
\hline Case & $\begin{array}{c}\text { Cost } \\
\text { (SEK/t slabs) }\end{array}$ & $\begin{array}{c}\mathrm{CO}_{2} \\
\text { (t/t slabs) }\end{array}$ \\
\hline Reference Case & 1409 & 1.936 \\
Case 1 & 1365 & 1.892 \\
Case 2 & 1461 & 1.994 \\
Case 3 & 1357 & 1.887 \\
Case 4 & 1359 & 1.884 \\
\hline
\end{tabular}

Comparison of the different cases with the reference regarding the cost objective is illustrated in Table 4. The results show that the cost optimization case followed by the case 1, where surface cleaning is utilized for the reference case, is generating the lowest production costs. The highest costs are shown to be generated in case 2, where no briquettes are produced. This shows that the use of briquettes and surface cleaning of scrap improves the cost analysis. In Cases 3 and 4, the cost decreases approximately $4 \%$, compared to the reference case. However the model only considers raw material cost related directly to the process and does not consider investment cost, salary, administration and other external costs. Furthermore no credits are made in the model for decreased landfill costs. Analysis of the results from using $\mathrm{CO}_{2}$ emission as the object function demonstrates only minor differences between the cases, as seen in Table 4. The case with the highest $\mathrm{CO}_{2}$ emission is Case 2 where no by-product briquettes are used; consequently an increased pellet and coke consumption generate higher $\mathrm{CO}_{2}$ emissions.

\subsection{Conclusions}

The system model shows the interaction between different processes, which gives an overview picture of the whole steel making route. The model is used to investigate changes in process conditions from making use of shredded scrap and ASR as input material in the steel making process. The system analysis shows that surface cleaning of scrap makes it possible to use shredded scrap and ASR or other combustible waste to replace fossil fuels as a cost and environmental efficiency choice. The implementation of surface cleaning and preheating leads to both increased possibilities for recycling materials, which otherwise is difficult to recycle and a more efficient use of energy in the steelmaking routes. Increased recycling of materials is efficient ways to reduce the energy demand, since it often replace virgin materials. The optimization of the model shows that both cost and $\mathrm{CO}_{2}$ can be decreased when scrap pretreatment is implemented, however none of the analyzed cases is simultaneous minimizing cost and $\mathrm{CO}_{2}$ emission.

\section{Acknowledgement}

This work is part of the ongoing projects in the Centre for Process Integration in Steelmaking (PRISMA) for the possibility to present this work. PRISMA is an Institute Excellence Centre supported by the Swedish Agency for Innovation Systems, the Knowledge Foundation, and eight industrial partners within the iron- and steel industry. This study has also received funding form the SSF ProInstitute programme. 


\section{References}

[1] M. Larsson, S. Ångström, A novel process for simultaneous scrap preheating and surface rinsing, SCANMET III, $3^{\text {rd }}$ International Conference on Process Development in Iron and Steelmaking, June 8-11, 2008, Luleå, Sweden.

[2] J. K. S. Tee, D.J. Fray, Recycling of galvanized steel scrap using chlorination, Iron and Steelmaking, 2005, Vol. 32, No 6, pp. $509-514$.

[3] K. Nilsson, M. Söderström, Optimizing the Operating Strategy of A Pulp and Paper Mill using the MIND Method, Energy - The International Journal, 1992, Vol. 17, No. 10, pp. 945-953

[4] M. Larsson, J. Dahl, Reduction of the specific energy use in an integrated steel plant- The effect of and optimisation model, ISIJ International, 2003, Vol. 43, No. 10, pp. 16641673

[5] C. Ryman, M. Larsson, Reduction of CO2-emissions from integrated steel-making by optimized scrap strategies: Application of process integration models on the BF-BOF system, ISIJ International, 2006, Vol. 46, No. 12, pp. 1752-1758

[6] C. Ryman, M. Larsson, Adaption of process integration models for minimization of energy use, CO2-emissions and raw material cost for integrated steelmaking, Chemical Engineering Transactions, 2007, Vol. 12, pp. 495 - 500.

[7] C. Ryman, C. Wang, M. Larsson, J. Dahl, C-E. Grip, Minimization of energy consumption and conversion cost for BF-BOF system based on optimized use of ferrous burden materials, $5^{\text {th }}$ European Oxygen Steelmaking Conference, June 26-28, 2006, Aachen, Germany.

[8] C. Wang, M. Larsson, C. Ryman, C-E Grip, J-O. Wikström, A. Johnsson, J. Engdahl, A model on $\mathrm{CO} 2$ emission reduction integrated steelmaking by optimization methods, International journal of energy research, 2008, 32, pp. 1092-1106

[9] U.S. Department of Energy, Energy Efficiency and Renewable energy, Steel Industry Technology Roadmap. http://www1.eere.energy.gov/industry/steel/roadmap.html (accessed during Jan. 2010).

[10]F. Su, S. Rutqvist, K. Andersson, Recycling of iron-bearing sludge and technological development at SSAB Tunnplat, CSM 2007 Annual Meeting Proceedings, November 1517, 2007, Chengdu City, China. 